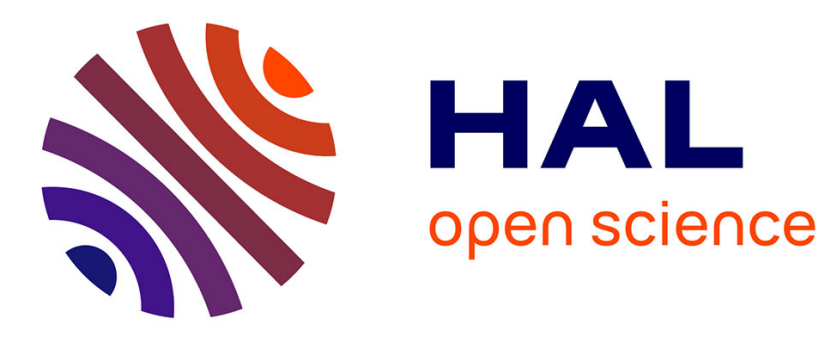

\title{
Phason dynamics in charge and spin density waves
}

\author{
A. Virosztek, K. Maki
}

\section{To cite this version:}

A. Virosztek, K. Maki. Phason dynamics in charge and spin density waves. Journal de Physique IV Proceedings, 1993, 03 (C2), pp.C2-203-C2-208. 10.1051/jp4:1993241 . jpa-00251324

\section{HAL Id: jpa-00251324 https://hal.science/jpa-00251324}

Submitted on 1 Jan 1993

HAL is a multi-disciplinary open access archive for the deposit and dissemination of scientific research documents, whether they are published or not. The documents may come from teaching and research institutions in France or abroad, or from public or private research centers.
L'archive ouverte pluridisciplinaire HAL, est destinée au dépôt et à la diffusion de documents scientifiques de niveau recherche, publiés ou non, émanant des établissements d'enseignement et de recherche français ou étrangers, des laboratoires publics ou privés. 


\title{
Phason dynamics in charge and spin density waves
}

\section{A. VIROSZTEK and K. MAKI ${ }^{*}$}

Research Institute for Solid State Physics, P.O. Box 49, 1525 Budapest, Hungary

* Department of Physics and Astronomy, University of Southern California, Los Angeles, CA 90089, U.S.A.

\begin{abstract}
Phason propagators in charge and spin density waves in presence of long range Coulomb interaction are studied within mean field theory. In charge density wave the longitudinal phason splits into an acoustic mode and an optical mode in the presence of Coulomb interaction. Except at low temperatures $\left(T \leq 0.2 T_{c}\right)$ the acoustic mode exhausts most of the optical weight. In spin density wave there is no longitudinal acoustic mode; the phason becomes the plasmon. On the other hand in the transverse limit, the plasmon decouples completely from the phason both in charge and spin density waves.
\end{abstract}

\section{Introduction.}

It is well known that the long range Coulomb interaction will modify the longitudinal phason mode strongly [1]. However, most works published on this subject [2] are incomplete due to unnecessary approximation. We shall report here our recent study of the phason dynamics [3] of both charge and spin density waves (CDW and SDW). As a model we take a quasi-one dimensional system (Fröhlich Hamiltonian [4] for CDW and Yamaji model [5] for SDW) supplemented by the long range Coulomb interaction

$$
H_{c}=4 \pi e^{2} \sum_{q} \frac{1}{q^{2}} n_{q} n_{-q}
$$

where $n_{q}$ is the electron density with momentum $\vec{q}$. As we shall see the condensate density $f$, which depends both on $\omega$ the frequency and $\vec{q}$ the momentum plays the crucial role in the following analysis. In the longitudinal limit (i.e. $\vec{q}$ parallel to the most conducting direction) we obtain an acoustic mode with the phason velocity decreasing rapidly with increasing temperature in CDW while there will be no phason in SDW. In particular this phason mode is observed recently by neutron scattering for a single crystal of $\mathrm{K}_{0.3}$ $\mathrm{MoO}_{3}$ [6]. In the transverse limit, on the other hand, the Coulomb effect disappears completely from the phason propagator in contrary to an early analysis of the electric conductivity in SDW [7].

\section{Phason Propagator in CDW.}

We shall first consider the phason propagator in CDW. In the presence of the long range Coulomb interaction the phason propator is given by

$$
D_{\phi}(\vec{q}, \omega)=\left(1-g^{2} \omega_{Q}^{2}\left(\omega_{Q}^{2}-\omega^{2}\right)^{-1}\left\langle\left[\Delta_{2}, \Delta_{2}\right]\right\rangle\right)^{-1} \omega_{Q}^{2}\left(\omega_{Q}^{2}-\omega^{2}\right)^{-1}
$$




$$
\begin{gathered}
=-(2 \Delta)^{2}(\lambda f)^{-1}\left(\frac{m^{*}}{m} \omega^{2}-\zeta^{2}-\omega_{p}^{2} f\left(1-\frac{\omega_{p}^{2}(1-f)}{\omega^{2}-\zeta^{2}}\right)^{-1}\right)^{-1} \\
\cong-(2 \Delta)^{2}(\lambda f)^{-1}(1-f)\left[\left(\frac{m^{*}}{m}(1-f)+f\right) \omega^{2}-\zeta^{2}\right]^{-1}
\end{gathered}
$$

where $\Delta_{2}$ is the imaginary part of the order parameter and the effect of the Coulomb interaction is incorporated within mean field theory [8]. Here we limit ourselves to the longitudinal case and in the last step we took $\omega_{p}=\infty$, where $\omega_{p}=\left(4 \pi e^{2} n / m\right)^{1 / 2}$ is the plasma frequency. The function $f$ is the generalized condensate density given by

$$
f=\left\{\begin{array}{l}
-\frac{1}{2} \int_{-\infty}^{\infty} d \phi \operatorname{th}\left(\frac{1}{2} \beta \Delta \operatorname{ch} \phi\right)\left[\operatorname{sh}^{2}\left(\phi-\phi_{0}\right)-\left(1-\alpha^{2}\right)(\zeta / 2 \Delta)^{2}\right]^{-1} \text { for } \alpha \leq 1 \\
\frac{1}{2} \int_{-\infty}^{\infty} d \phi \operatorname{th}\left(\frac{1}{2} \beta \Delta \operatorname{ch} \phi\right)\left[\operatorname{ch}^{2}\left(\phi-\phi_{0}\right)+\left(1-\alpha^{2}\right)(\zeta / 2 \Delta)^{2}\right]^{-1} \text { for } \alpha>1
\end{array}\right.
$$

where $\alpha=\omega / \zeta=\omega / v q$ and

$$
\text { th } \phi_{0}= \begin{cases}\alpha & \text { for } \alpha \leq 1 \\ \alpha^{-1} & \text { for } \alpha>1\end{cases}
$$

We recover familiar expressions in the adiabatic limit $(\omega, \zeta \ll 2 \Delta(T))$

$$
f_{s}=\lim _{\alpha \rightarrow 0} f=2 \pi T \sum_{n=0}^{\infty} \frac{\Delta^{2}}{\left(\omega_{n}^{2}+\Delta^{2}\right)^{3 / 2}}
$$

and

$$
f_{d}=\lim _{\alpha \rightarrow \infty} \int_{0}^{\infty} d \phi \operatorname{sech}^{2} \phi \operatorname{th}\left(\frac{1}{2} \beta \Delta \operatorname{ch} \phi\right)
$$

Also $f=1$ for $\alpha=1$ independent of temperature. The temperature dependence of $f_{s}$ and $f_{d}$ are shown in Fig. 1. The phason mass $m^{*}$ is given by [9]

$$
\frac{m^{*}}{m}=1+(2 \Delta)^{2}\left(\lambda \omega_{Q}^{2} f\right)^{-1}
$$

It is important to note that the phason mass $m^{*}$ has different temperature dependence depending on which limit you are in. As already pointed out by Takada and his collaborators [2], the longitudinal phason consists of 2 modes, which is determined from

$$
\left(1+\frac{(2 \Delta)^{2}}{\lambda \omega_{Q}^{2}}\left(f^{-1}-1\right)\right) \omega^{2}=\zeta^{2}
$$

where substituted $\mathrm{Eq}(7)$ in the pole of $\mathrm{Eq}(2)$ 


\section{Acoustic Mode.}

One of the solutions is given by

$$
\omega^{2}=\frac{m}{m^{*}}\left(1-f+\frac{m}{m^{*}} f^{-1} \zeta^{2}\right.
$$

where $m^{*} / m$ defined in Eq (7) has to be used. Then for not too low temperatures (say $T>0.3 T_{c}$ ) we have $\omega \ll \zeta$ and $\mathrm{Eq}(9)$ simplifies as $\omega=v_{\phi} q$ with

$$
v_{\phi} / v_{F}=\left(\frac{m}{m_{s}^{*}}\right)^{\frac{1}{2}}\left(1-f_{s}+\frac{m}{m_{s}^{*}} f_{s}\right)^{-\frac{1}{2}}
$$

where suffix $s$ means the static limit $(\alpha \ll 1)$. The temperature dependence of $v_{\phi}$ is shown in Fig. 2 . As seen from Fig. $2, v_{\phi}$ increases rapidly with indecreasing temperature and ultimately it merges with another

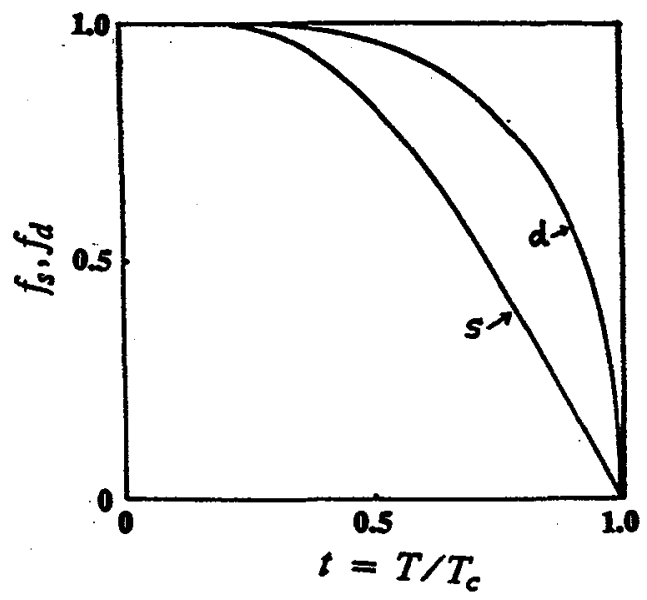

Fig. 1

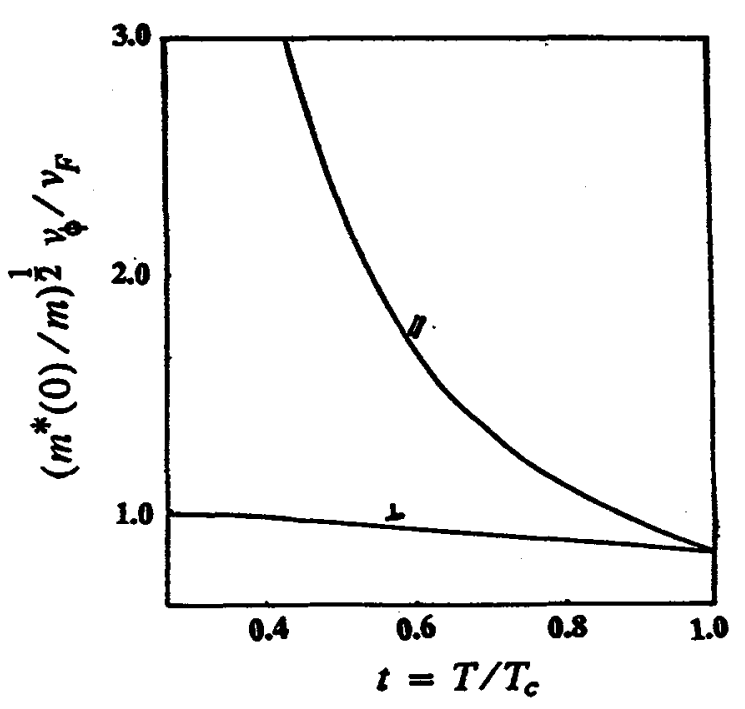

Fig. 2

Fig. 1. - The condensate density $f_{s}$ (the static limit) and $f_{d}$ (the dynamic limit) are shown as function of the reduced temperature $t$.

Fig. 2. - The longitudinal and the transverse phason velocities $v_{\phi w}$ and $v_{\phi_{\perp}}$ in CDW are shown as function of reduced temperature.

mode and disappears at low temperatures $\left(T \cong 0.2 T_{c}\right)$. It is of interest to consider the spectral weight. Total spectral weight of phason is given from $\mathrm{Eq}(2)$ as

$$
\int_{0}^{\infty} d \omega \omega \operatorname{Im} D_{\phi}(q, \omega)=\frac{\pi}{2} \omega_{Q}^{2}
$$

independent of $\zeta, T$ and $\omega_{p}$. The acoustic pole gives, on the other hand, 


$$
\int_{0}^{\infty} d \omega \omega \operatorname{Im} D_{\phi}(q, \omega)=\frac{\pi}{2} \omega_{Q}^{2}\left(\frac{1-f_{s}}{1-f_{s}+\frac{m}{m_{s}^{*}} f_{s}}\right)\left(1-\frac{m}{m_{s}^{*}}\right)
$$

which almost exhausts the spectral weight as long as $1-f_{s} \gg m / m_{s}^{*}$ (i.e. $T \geq 0.3 T_{c}$ ).

For an arbitrary $\vec{q}$ the acoustic mode is given by

$$
\omega \cong\left(\frac{m}{m_{s}^{*}}\right)^{\frac{1}{2}}\left(1-f_{s}\right)^{-\frac{1}{2}}\left(\zeta_{\mu}^{2}+\left(1-f_{s}\right) \zeta_{\perp}^{2}\right)
$$

where $\zeta_{\nu}=v q_{n}$ and $\zeta_{\perp}=v_{\perp} q_{\perp}$ and $v_{\perp}$ is the Fermi velocity in the transverse direction.

In the transverse limit (i.e. $\zeta_{\| \prime}=0$ ) where the Coulomb interaction is completely decoupled, the phason velocity $v_{\phi \perp}$ depends only weakly on $T$ through $m_{s}^{*}(T)$ as shown in Fig. 2.

\section{Optical Mode.}

At $T=0 K$ another solution of $\mathrm{Eq}(8)$ is [2]

$$
\omega_{o p}^{2}=\frac{3}{2} \lambda \omega_{Q}^{2}+\frac{1}{5} \frac{m}{m^{*}} \zeta^{2}
$$

The optical frequency is almost independent of $T$ for $T<0.2 T_{c}$ and then start to increase with increasing temperature. At the same time the optical weight of this mode decreases very rapidly. At $T=0 K$, the optical mode almost exhausts the optical weight.

\section{Phason Propagator in SDW.}

Since $m * / m=1$ practically for SDW, the phason propagator is now given by

$$
D_{\phi}(q, \omega)=(2 \Delta)^{2}\left\{\left\langle f\left(\zeta^{2}-\omega^{2}\right)\right\rangle+\frac{4 \pi e^{2}}{q^{2}} N_{o}\langle f \zeta\rangle^{2}\left[1+\frac{4 \pi e^{2}}{q^{2}} N_{o}\left\langle\frac{\zeta^{2}}{\zeta^{2}-\omega^{2}}(1-f)\right\rangle\right]^{-1}\right\}^{-1}
$$

where \langle\rangle means the angular average and

$$
\zeta=\zeta_{\mu}+\sqrt{2} \zeta_{\perp} \cos \phi
$$

In the longitudinal limit $\left(\zeta_{\perp}=0\right) \mathrm{Eq}(15)$ reduces to

$$
D_{\phi}(q, \omega)=(2 \Delta)^{2} \bar{f}^{-1}\left(\omega_{p}^{2}(1-f)+\zeta^{2}-\omega^{2}\right)\left(\zeta^{2}-\omega^{2}\right)^{-1} \times\left(\omega_{p}^{2}+\zeta^{2}-\omega^{2}\right)^{-1}
$$

Since $f_{\omega= \pm \zeta}=1, D_{\phi}(\vec{q}, \omega)$ has only the plasmon pole in the longitudinal limit. More generally in the limit $\omega \gg \zeta \mathrm{Eq}(15)$ can be rewritten as

$$
D_{\phi}(q, \omega)=(2 \Delta)^{2} \vec{f}^{-1}\left(1-\frac{\omega_{p}^{2}}{\omega^{2}}\left(\cos ^{2} \vartheta+\left(\frac{v_{\perp}}{v_{*}}\right)^{2} \sin ^{2} \vartheta\right)(1-f)\right) \times
$$




$$
\left[\omega_{p}^{2}\left(\cos \vartheta+\left(\frac{v_{\perp}}{v_{\prime \prime}}\right)^{2} \sin ^{2} \vartheta(1-f)\right)-\omega^{2}\right]^{-1}
$$

where $\cos \vartheta=q_{1 \prime} / q$. The pole is then given by

$$
\omega^{2}=\omega_{p}^{2}\left\{\cos \vartheta+\left(\frac{v_{\perp}}{v_{\prime \prime}}\right)^{2} \sin ^{2} \vartheta(1-f(\omega))\right\}
$$

which always has two solutions. One at high frequencies (i.e. $\omega \gg 2 \Delta(T)$ )

$$
\omega_{+}^{2}=\omega_{p}^{2}\left\{\cos \vartheta+\left(\frac{v_{\perp}}{v_{\prime \prime}}\right)^{2} \sin ^{2} \vartheta\right\}
$$

and another below the quasi-particle energy gap;

$$
\omega_{-}^{2}=\frac{3}{2}(2 \Delta)^{2}\left(1-\frac{3}{2} G_{d}\right)^{-1}\left(1-f_{d}+\left(\frac{\zeta_{\prime \prime}}{\zeta_{\perp}}\right)^{2}\right)
$$

where $f_{d}$ is the condensate density in the dynamical limit and

$$
G_{d}=2 \int_{0}^{\infty} d \phi \operatorname{sech}^{4} \phi\left(1+e^{\beta \Delta \operatorname{ch} \Phi}\right)^{-1}
$$

At $T=0 K$ where $f_{d}=1$, the second mode is almost gapless when $\zeta_{\text {, }} \ll \zeta_{\perp}$. In deriving Eq (21) it was assumed $\left(\omega_{-} / 2 \Delta\right) \ll 1$. In general the optical weight is dominated by the $\omega_{-}$mode. However, when the longitudinal limit is approached $\left(\zeta_{\perp} \rightarrow 0\right), \omega_{-}$becomes $2 \Delta$ and the $\omega_{-}$mode loses the optical weight completely and the optical weight shifts completely to the $\omega_{+}$mode. Also as temperature increases (say $T$ $\geq 0.2 T_{c}$ ), $\omega_{-}$increases rapidly and again the present approximation breaks down completely. In the transverse limit $\left(\zeta_{\prime \prime}=0\right)$ on the other hand, the Coulomb potential drops out completely and we recover the old result [10]

$$
D_{\phi}(\vec{q}, \omega)=(2 \Delta)^{2} f^{-1}\left(\zeta_{\perp}^{2}-\omega^{2}\right)^{-1}
$$

\section{Concluding Remarks.}

We have shown that the long range Coulomb interaction has dramatic effect on the phason propagator in both CDW and SDW. In CDW the phason velocity in the longitudinal limit $v_{\phi}$ becomes strongly temperature dependent as observed in a recent neutron scattering experiment in a single crystal of blue bronze $\mathrm{K}_{0.3} \mathrm{MoO}_{3}$ [6]. At low temperatures the Coulomb interaction generates an optical mode, which has not been seen experimentally. In SDW the acoustic mode disappears in the longitudinal limit. At low temperatures $\left(T \leq 0.2 T_{c}\right)$ there will be an optical mode with small energy gap. On the transverse limit the Coulomb interaction effect disappears completely and we recover the old results [10] without Coulomb interaction 


\section{Acknowledgements.}

One of us (KM) thanks the Research Institute for Solid State Physics at Budapest for the kind hospitality where a part of this work was done. The present work is supported by National Science Foundation under Grant No DMR 92-18317, the Hungarian National Research Fund No. OTKA 2944 and T4473. Also this work is sponsored by the US - Hungary Science and Technology Joint Fund under Project No 264.

\section{References}

[1] LEE P.A. and FUKUYAMA H., Phys. Rev. B 17 (1987) 542.

[2] NAKANE Y. and TAKADA S., J. Phys. Soc. Jpn. 54 (1985) 977; WONG K.Y. and TAKADA S., Phys. Rev. B 36 (1987) 5476.

[3] VIROSZTEK A. and MAKI K., Phys. Rev. Lett. 69 (1992) 3265; Synth. Metals 57 (1993) 4678 and Phys. Rev. B 48 (1993).

[4] LEE P.A., RICE T.M. and ANDERSON P.W., Solid State Commun 14 (1974) 703.

[5] YAMAЛ K., J. Phys. Soc. Jpn. 51 (1982) 2787 and 52 (1983) 1361.

[6] HENNION B., POUGET J.P. and SATO M. Phys. Rev. Lett. 68 (1992) 2374.

[7] MAKI K. and GRUNER G., Phys. Rev. Lett. 66 (1991) 782.

[8] KADANOFF L.P. and FALKO I.I., Phys. Rev. 136 (1964) 1170.

[9] MAKI K. and VIROSZTEK A., Phys. Rev. B 36 (1987) 511.

[10] VIROSZTEK A. and MAKI K., Phys. Rev. B 37 (1988) 2028; MAKI K. and VIROSZTEK A., Synth. Metals 55 (1993) 2774. 